\title{
practice
}

\section{Peripheral arterial disease in patients with renal-diabetic foot ulcers}

Objective: To describe the angiographic characteristics of peripheral arterial disease (PAD) in persons with diabetic foot ulcers (DFUs) on dialysis treatment.

Method: The study is a retrospective analysis of patients with DFUs and PAD who had been referred to our diabetic foot clinic. All patients had been managed by a pre-set limb salvage protocol including revascularisation of the affected limb. Arterial lesions (stenosis between $50-99 \%$ and occlusions) were retrospectively evaluated through angiogram analysis. According to the presence or not of dialysis, patients were divided into two patient groups: renal-diabetic foot (RDF) and diabetic foot (DF). Distribution of PAD and immediate revascularisation outcome (technical revascularisation outcome) for RDF and DF were separately reported and compared.

Results: The sample included 239 patients: mean age was 71.8 years; $72.4 \%$ were male; $87.4 \%$ had type 2 diabetes; mean diabetes duration was 21.4 years; and the mean $\mathrm{HbA} 1 \mathrm{c}$ was $63 \pm 22 \mathrm{mmol} / \mathrm{mol}$. The RDF group compared with the DF group reported higher numbers of vessels affected $(n=5 \pm 1.6$ versus $3.9 \pm 1.5$, respectively, $p<0.0001$ ), greater involvement of the superficial femoral artery $(90.2 \%$ versus $75.8 \%$, respectively, $\mathrm{p}=0.003)$, the tibial-peroneal trunk ( $53.7 \%$ versus $25.5 \%$, respectively, $p=0.01)$, the anterior tibial artery $(93.9 \%$ versus $80.9 \%$, respectively, $\mathrm{p}=0.03$ ) and below-the-ankle (BTA) arteries ( $70.7 \%$ versus $35.7 \%$, respectively, $p=0.0001)$. The RDF group showed a higher rate of revascularisation failure in comparison to DF patients $(43.9 \%$ versus $15.3 \%$, respectively, $\mathrm{p}<0.0001)$. BTA arterial disease (odds ratio 9.5; 95\% Confidence Interval: 3.5-25.4; $\mathrm{p}=0.0001$ ) resulted as the only independent predictor of revascularisation failure.

Conclusion: In this study, RDF patients showed a widespread distribution of arterial lesions with a higher involvement of foot arteries in comparison with DF patients. BTA arterial disease was found to be an independent predictor of revascularisation failure. Declaration of interest: The authors have no conflicts of interest.

arterial lesions $\bullet$ diabetes $\bullet$ diabetic foot ulcer $\bullet$ dialysis $\bullet$ end-stage renal disease $\bullet$ limb salvage $\bullet$ peripheral arterial disease $\bullet$ renal $\bullet$ revascular

\section{Pagina $1 / 5 \quad+\quad+$}

